\title{
NOTICE EXPLICATIVE
}

La Liste mondiale des Universités a été conçue comme un répertoire succinct susceptible de faciliter les échanges à travers le monde de l'enseignement supérieur dans son ensemble. Elle comprend deux parties: la première présente pays par pays des renseignements sur les universités et autres établissements d'enseignement supérieur, ainsi que sur les organismes universitaires et étudiants; la deuxième traite des organisations internationales et régionales s'occupant d'enseignement supérieur.

\section{PREMIERE PARTIE}

\section{Etablissements et Organisations Nationales}

Les renseignements sont classés par pays et territoires (au nombre de 154). Ceux-ci sont présentés dans l'ordre alphabétique de leur nom en anglais, mais un double index, anglais et français, est inclus (pages 605-610).

Chaque chapitre commence par une liste des universités et autres établissements d'enseignement supérieur dans le pays ou territoire intéressé, puis viennent des renseignements sur les organismes universitaires et étudiants nationaux.

(a) Universités et autres institutions d'enseignement supérieur

Les termes «université» et «institution d'enseignement supérieur» ont, on le sait, un contenu quelque peu variable selon les pays. La sélection des institutions figurant dans la Liste mondiale des Universités a été opérée principalement en fonction de l'usage des organismes académiques ou d'éducation des pays intéressés.

Pour les pays comptant un grand nombre d'institutions, celles-ci sont en règle générale réparties en deux rubriques principales:

(i) Universités (y compris, le cas échéant, les universités techniques et autres établissements pluridisciplinaires jouissant pleinement du statut universitaire;

(ii) Autres institutions d'enseignement supérieur.

Dans la plupart des cas, la seconde rubrique se trouve elle-même subdivisée en établissements d'enseignement technique; d'enseignement professionnel; de formation pédagogique; et d'enseignement général.

Cette classification n'a été adoptée que pour la commodité des usagers de la Liste mondiale des Universités et ne doit nullement être considérée comme une tentative "d'évaluation» de l'Association internationale des Universités. Il convient de noter que les écoles ou facultés indépendantes de théologie n'ont pas été mentionnées, non plus que les écoles de formation militaire.

On trouvera pour chaque institution les renseignements suivants: son nom (assorti, le cas échéant, de sa traduction anglaise ou française), son 
adresse et sa date (réelle ou légale) de fondation. Dans certains cas, deux dates ou plus sont indiquées: la première est la date primitive de fondation; les suivantes celles auxquelles des modifications importantes sont intervenues dans le statut de l'institution, comme, par exemple, un collège fondé en 1917, et qui a reçu le statut d'université en 1936.

Pour chaque institution, il est également fait mention, s'il y a lieu, de sa composition par facultés, collèges, écoles, départements ou instituts. A cette fin, des abréviations sont utilisées pour les termes tant anglais que français et on en trouvera le code, dans les deux langues, aux pages xvi-xxi. Ces indications ne font qu'indiquer le caractère général de l'établissement et n'embrassent pas nécessairement toutes les matières principales d'enseignement. C'est ainsi qu'une faculté de médecine peut ou non comporter une section d'odontologie, de pharmacie ou une école d'infirmières; une faculté de philosophie englobant les humanités peut ou non enseigner les sciences sociales. On trouvera à ce sujet des renseignements détaillés dans une autre publication de l'Association internationale des Universités, l'International Handbook of Universities, et dans l'ouvrage dont il constitue un complément, le Commonwealth Universities Yearbook, publié par l'Association of Commonwealth Universities, Londres. Des descriptions des systèmes universitaires figurent dans un autre ouvrage de reférénce préparé par l'Association internationale des Universités pour l'Unesco: Les études supérieures: présentation comparative des régimes d'enseignement et des diplômes, Unesco (deuxième édition en préparation).

Le titre officiel de la personne à laquelle il convient habituellement d'adresser la correspondance générale (Secrétaire général, Registrar, etc.), est également indiqué pour les institutions universitaires. Il est donné pour l'université figurant en tête de la liste de chaque pays, et sauf indication contraire, il reste le même pour toutes les autres universités du pays considéré.

Les institutions marquées d'un astérisque (*) ont été admises à la qualité de membre de l'Association internationale des Universités avant le $1^{\text {er }}$ octobre 1982 (Voir aussi pp. 581-594).

\section{(b) Organismes nationaux universitaires et étudiants}

La deuxième section de chaque chapitre répertorie les associations d'universités, les conférences ou comités de recteurs et de vicechanceliers, les associations d'enseignants universitaires, les bureaux d'information universitaire et les associations ou unions d'étudiants. Les services gouvernementaux (en général les ministères de l'éducation) qualifiés pour donner des renseignements en matière d'enseignement supérieur, ainsi que les Commissions nationales pour l'Unesco, ont été indiqués à la fin de la rubrique consacrée à chaque pays ou territoire. Les sociétés savantes, les organismes scientifiques et les associations académiques s'occupant de disciplines particulières, par contre, ne sont pas répértoriés.

Nombre de ces organismes s'emploient à faciliter les échanges universitaires, assurent des services aux enseignants, chercheurs et étudiants étrangers désireux de venir dans leurs pays respectifs ou apportent leur aide aux enseignants, chercheurs et étudiants nationaux désireux de travailler dans des universités étrangères. Dans les quelques pays où aucun organisme de ce genre n'est mentionné, on pourra s'adresser directement aux universités elles-mêmes ou au ministère de l'éducation.

On trouvera des notes descriptives sur un grand nombre des organisa- 
tions principales mais non, en règle générale, sur celles qui se rattachent aux organismes internationaux dont il est traité plus en détail dans la deuxième partie de l'ouvrage. Lorsque l'anglais est la langue du pays intéressé ou la langue étrangère la plus usitée, la note descriptive figure d'abord en anglais, suivie par une traduction française en italique. Inversement, la note paraît d'abord en français dans les cas où cette langue prédomine, et est alors suivie d'une traduction anglaise en italique. Là où les organismes internationaux ont indiqué les adresses de leurs sections ou adhérents nationaux, ceux-ci figurent sous les pays intéressés, leur lien avec une organisation internationale étant marqué par l'adjonction à leur nom du sigle de celle-ci: par ex. Associazione nazionale Professori universitari di Ruolo (IAUPL).

Université des Nations Unies

Institut Universitaire Européen

La dernière section de la première partie continent des renseignements sur l'Université de Nations Unies et l'Institut Universitaire Européen.

\section{DEUXIEME PARTIE}

Cette partie constitue un guide des principales organisations internationales et régionales s'occupant principalement d'enseignement supérieur. Elle comprend des rubriques consacrées tant à l'Association internationale ellemême qu'à ses huit membres associés, ainsi qu'une rubrique traitant de l'œuvre de l'Unesco dans ce domaine. Des notes descriptives, données tant en anglais qu'en français, passent brièvement en revue l'histoire, les buts, la structure et les activités de chaque organisme; les textes de ces notes ou les renseignements qui y figurent ont été fournis par les organismes eux-mêmes. Un certain nombre d'organisations internationales s'occupant de certaines activités particulières concernant les étudiants sont indiquées plus brièvement. Mais, pas plus que dans les chapitres consacrés aux différents pays, on n'a répertorié les sociétés savantes, les organismes scientifiques ou les associations académiques s'occupant de disciplines particulières. 